\title{
Microstructural change in ice: III. Observations from an iceberg impact zone
}

\author{
K. J. Muggeridge, ${ }^{*}$ I. J. JordaAn \\ Ocean Engineering Research Centre, Faculty of Engineering and Applied Science, Memorial University of. Newfoundland, \\ St fohn's, Nerefoundland AIB 3X.5, Canada
}

\begin{abstract}
During a full-scale iceberg-impact study conducted in July 1995 on the Labrador coast, Canada, a sample of ice was retrieved from the impacted surface of an iceberg. The sample was thin-sectioned and the observations of the contact-zone microstructure are presented in this paper.

Thin sections were prepared from two slabs cut parallel to the impacted surface. In each of the thin sections taken from the impacted-surface slab, fine-grained material was found to surround parent-size grains (as observed in the second slab). A boundary between the parent grains and the grains of modified microstructure was found running approximately parallel to the impacted surface in each of the thin sections taken from the impacted-surface slab. This boundary was pronounced towards the edges of the contact zone. Lateral movement of grains outward along this boundary was observed in thin sections near the edges but not near the centre of the contact zone.

The thin sections were compared to the results of medium-scale indentation tests in 1989 and 1990 from the Arctic Ocean. The same type of fine-grained material and layer formation of modified microstructure was found in the contact zones.
\end{abstract}

\section{INTRODUGTION}

During summer 1995, full-scale iceberg-impact tests were conducted on Grappling Island (near Cartwright), Labrador, Canada, by the Centre for Cold Ocean Resources Engineering (C-CORE), Memorial University of Newfoundland, and K. R. Croasdale and Associates Ltd (Crocker and others, 1997). The aim of this field test program was to measure the loads generated during the impact of towed icebergs against a fixed instrumented panel. The need for full-scale testing of iceberg impacts was recognized in the early $1980 \mathrm{~s}$, because uncertainties associated with extrapolating ice-crushing strength measurements from small-scale laboratory tests to full-scale offshore structures led to conservative and thus costly designs (Crocker, 1995).

The goal of this paper is to present the microstructural changes found in a sample taken from the contact zone of an impacted iceberg. A sample of ice containing a contact zone was collected following an impact test at the test site on Grappling Island. The sample was stored in a freezer, and later thin-sectioned in a controlled laboratory setting in St John's, Newfoundland. The microstructure of the contact zone is presented through a series of thin sections taken along the impacted surface of the contact zone. The thin sections show the variation in microstructure along the impacted surface of the contact zone. A second set of thin sections was taken from ice directly below the first slab of ice to observe the microstructure behind the centre and edge of the contact zone.

* Present address: C-CORE, Memorial University of Newfoundland, St John's, Newfoundland AIB 3X5, Canada.
The microstructural changes found in this contact zone are of interest for comparison with laboratory-tested specimens. This paper is presented as one of three companion papers with Melanson and others (1999) and Meglis and others (1999). These papers present some of the recent laboratory ice-test programs conducted at Memorial University of Newfoundland.

\section{BACKGROUND}

Development of the oil and gas industry operating off the east coast of Canada has been spurred by the recent Hibernia project, starting from the early exploration drilling conducted in the 1970s and early 1980s. The first oil has been recovered at the Hibernia site ahead of schedule, in November 1997, and the Terra Nova project is well underway. Other fields such as Whiterose, Bonne Bay, Hebron and Ben Nevis are expected to provide significant future discoveries. Oil and gas operations in the Grand Banks area require particular attention to environmental conditions in the area. These include icebergs and severe storm-generated wind and wave conditions. The consequences of iceberg impacts can be severe, and consideration must be given to the probability of these impacts and the associated loads based on the type of structure, location of production site and time of operations.

One of the main achievements of the ice group at Memorial University is the completion of a comprehensive model for estimating iceberg design loads for various types of production systems (Memorial University of Newfoundland, 1996). This includes estimation of global and local ice loads for different production systems at a number of probability of exceedance values. It was shown that design global and 
local loads could be reduced substantially compared to those previously used by industry, leading to more economical designs. The reduction is associated mainly with improved analysis of the ice-failure process. Past assessments have generally been based on a conservative design premise, for example using conservative estimates of ice pressure. As knowledge and understanding of the ice-failure processes improve, there is potential for making more realistic assessments, and for reducing uncertainty in the design-load estimates. Full-scale test data and information from impacted icebergs are key to obtaining a better understanding of the behavior of ice during impact.

The failure process of ice is complex, with a number of processes occurring during the interaction. Fractures in the form of spalls reduce the nominal area of contact, and highpressure zones are produced during the impact. High-pressure zones are areas of high pressure surrounded by areas of relatively low pressure (Jordaan and others, 1999). Highpressure zones accommodate large shear stresses as well as high confining pressures towards the centre of these zones. As the compressive failure occurs within the high-pressure zone, evidence of microcracking and pressure melting at the grain boundaries has been observed in the field during medium-scale indentation tests and small-scale laboratory tests. As the high-pressure zone(s) fail, recrystallization of grains and refreezing can occur. Small ice pieces are extruded at the free edges of the contact zone with the release of the confining pressure. Extrusion of fine ice particles has been observed in the field at the Molikpaq structure in the Beaufort Sea and during medium-scale indentation tests. A schematic of a high-pressure zone and the extrusion of crushed ice is shown in Figure 1. See Melanson and others (1999) for further discussion on high-pressure zones.

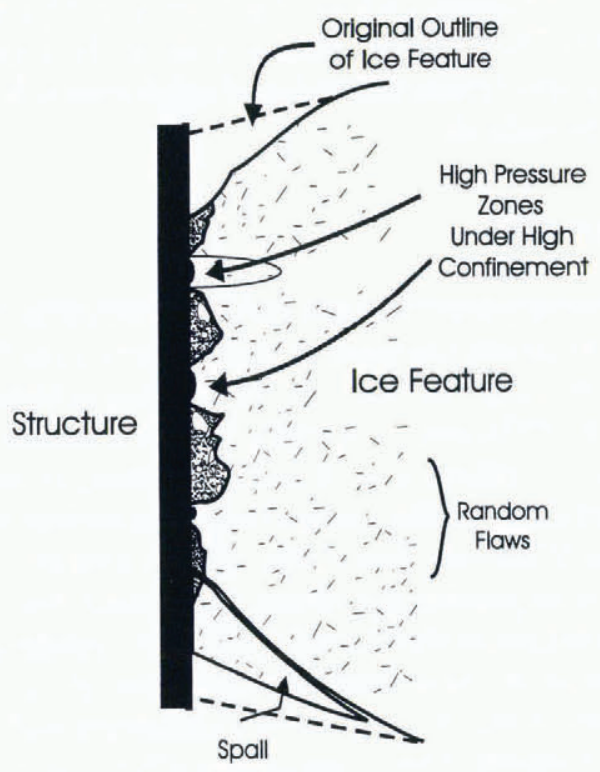

Fig. 1. Schematic of high-pressure zone.

\section{PROCEDURE}

\section{Impact experiment}

The test site was located on Grappling Island approximately $14 \mathrm{~km}$ by boat from Cartwright. A steel panel, measuring $6 \mathrm{~m}$ by $6 \mathrm{~m}$ and weighing approximately $30 \mathrm{t}$, was instrumented to measure impact loads. This panel was mounted on a near-vertical cliff face with most of the panel below the water surface. The panel consisted of 36 triangular subpanels, each of which had a single load cell located at every corner (a total of 108 load cells for the panel) capable of measuring a uniaxial load. A schematic of the test configuration is shown in Figure 2. A rope was attached to the iceberg, passed through a snatch block located just above the centre of the panel, and then connected to the towing vessel. A pendulum line was used to align the iceberg with the centre of the panel, as shown in Figure 2. Following impact, the pendulum line was released and the iceberg was towed into position for another impact or moved off site so that testing could proceed with a new iceberg. Because towing a single iceberg to the site often took several hours, each iceberg was typically impacted a number of times, providing it was still of significant size.

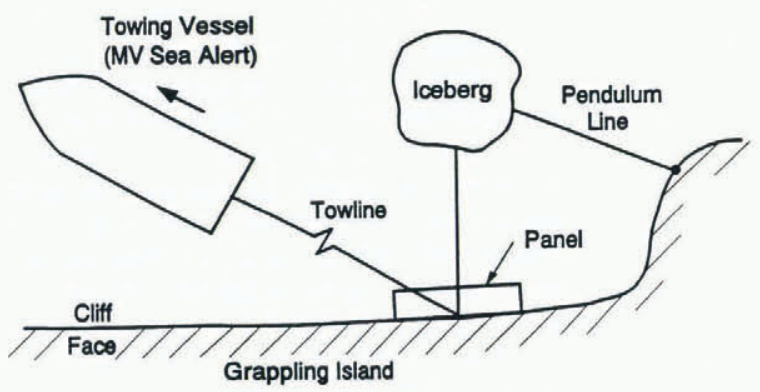

Fig. 2. Schematic of iceberg-impact test configuration (plan view).

A total of 30 iceberg impacts was recorded on the panel. The icebergs were in the range 500-1500t, with impact speeds reaching $1.5 \mathrm{~m} \mathrm{~s}^{-1}$ (Crocker, 1995). Photographs were taken of the icebergs as they impacted the panel. Plate $11^{*}$ shows the second impact of "Steve B", an iceberg with a mass of approximately $700 \mathrm{t}$ at the time of testing. Pieces of ice can be seen flying in the air quite some distance from the point of contact.

\section{Sample collection and preparation}

Icebergs generally impacted the panel well below the waterline, and their contact zones normally remained underwater. Samples could be retrieved only if the iceberg rolled and exposed the contact zone, which did not occur often. After the fifth impact of iceberg "Marlene", a contact zone was spotted above the surface of the water. Iceberg "Marlene" had a mass of approximately $500 \mathrm{t}$ at the time of testing. It is believed that the impact occurred with the cliff face rather than the panel, since the impacted surface was brown in colour with traces of marine plant life. For this reason, there are no load-time data available for this impact. The speed of the iceberg was approximately $1 \mathrm{~m} \mathrm{~s}^{-1}$. The ambient temperature was approximately $10^{\circ} \mathrm{C}$ and the water temperature approximately $5^{\circ} \mathrm{C}$. The temperature of the ice in the area of the contact zone is estimated to be between $-5^{\circ}$ and $-10^{\circ} \mathrm{C}$.

A block of ice containing the contact zone was extracted from iceberg "Marlene" using a chain-saw. The contact zone on "Marlene" is seen as a brown spot in Plate 12. The block of

\footnotetext{
* For colour plates see section before previous paper.
} 
ice was immediately transferred to a cooler, and was stored in a freezer for 3 days before being transported to Memorial University for microstructural analysis.

\section{Thin-section analysis of contact-zone material}

The sample was stored at $-30^{\circ} \mathrm{C}$ at Memorial University to prevent significant modification of the microstructure prior to preparation of thin sections approximately 3 weeks later. Thin sections were prepared from two slabs taken from the sample containing the contact zone. A schematic of the ice sample showing the contact zone and the numbered thin sections is given in Figure 3. The contact zone was approximately oval in shape, with the major axis measuring $160 \mathrm{~mm}$ and the minor axis $110 \mathrm{~mm}$. The ice sample was first cut parallel to the plane of the impacted surface to form a slab of ice approximately $80 \mathrm{~mm}$ thick. This slab was called the "impacted-surface" slab and denoted IS. The approximate centre line of the contact zone was marked to indicate the relative positions of the thin sections along the impacted surface. Thin sections numbered 10 through 1 , and la through ld, were taken from the impacted-surface slab as shown in Figure 3. A second slab, denoted slab 2 (S2), $80 \mathrm{~mm}$ thick was cut parallel to the first slab. The centre line of the impacted surface was maked slab 2, and the general alignment of thin sections taken from this slab in relation to those taken from the impacted-surface slab is shown in Figure 3. The labelling of thin sections starts with CZ1 (contact zone 1), followed by the slab, IS or S2, and the thin-section number. For example, thin section 8 from the impacted-surface slab is denoted CZI/IS/TS8.
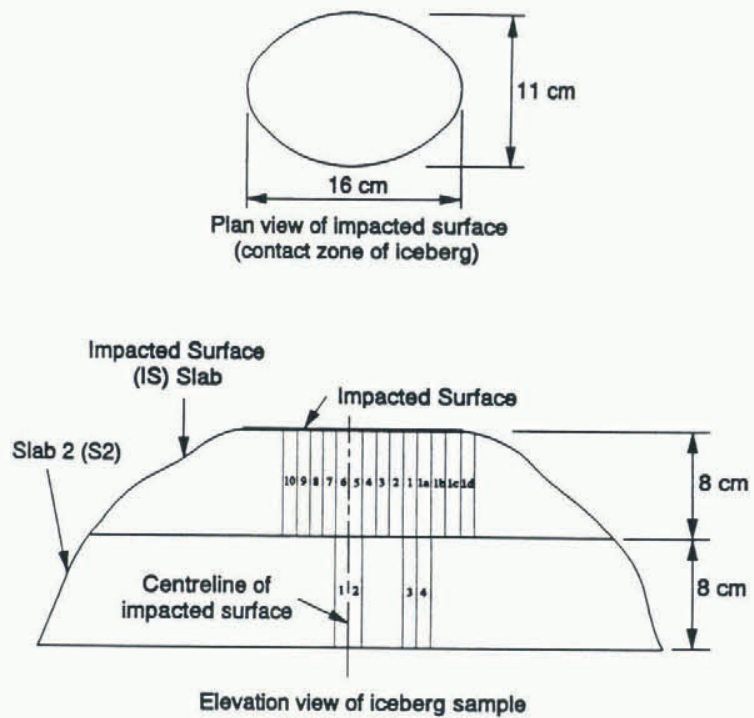

Fig. 3. Schematic of iceberg sample containing contact zone and position of thin sections.

All the thin sections were microtomed to approximately $1 \mathrm{~mm}$ thickness or less and then photographed between cross-polarized filters the same day they were prepared. Each section was originally cut with a band-saw to approximately $10 \mathrm{~mm}$ thickness. The microtoming process began by attaching the section to a glass plate with three or four drops of water applied along the perimeter of the thin section. The thin sections were microtomed until a smooth, level surface was obtained. They were then turned over on the glass plate so that the flat side of the thin section was against the glass plate. The thin section was attached to the glass plate with a continuous bead of water and then microtomed to a thickness of $<1 \mathrm{~mm}$. Thin sections 5 and 7 of the impacted-surface slab and thin section 2 of slab 2 were lost during the microtoming process. The thin sections from this sample were found to be extremely fragile, particularly the ones closest to the centre of the contact zone.

Each of the thin sections mentioned was photographed between cross-polarized filters at a number of standard magnifications. The orientation of the thin sections was such that the top of the photograph represented the top of the impacted surface for thin sections taken from the impacted-surface slab. The orientation of thin section 3 taken from slab 2 was inverted in the photograph. Global photographs of all thin sections were taken. A number of additional photographs were taken of each thin section at two magnification settings. A scale in $\mathrm{mm}$ is shown in all of the photographs.

A few additional photographs of selected thin sections were taken with plain light shone from the side to look at the predominant crack pattern. These photographs were taken approximately l year later. The thin sections were too fragile to microtome on both sides, so only the top side was microtomed. Even though the storage temperature was $-30^{\circ} \mathrm{C}$, some sublimation occurred between the thin section and the glass plate. Sample photographs have been included since the pattern of cracking can be easily seen. The other details in these photographs will be ignored, since they are readily visible in the photographs taken of the same thin sections directly after the original thin-sectioning process.

\section{OBSERVATIONS AND INTERPRETATION}

For brevity, only selected photographs are presented. Photographs of the global view of particular thin sections are shown in Plate 13. Magnified views of selected thin sections are shown in Plate 14. Selected photographs under plainand side-lighting conditions are shown in Plate 15. In all of the global views, the microstructure at the immediate edge of the thin sections corresponds to the frozen bead of water used to weld the thin section to the glass plate.

In the global views of the thin sections taken along the impacted surface of the contact zone (Plate 13a-d), finegrained material was found to be present in all of the thin sections. Thin sections taken from slab 2 (Plate l3e and f) indicate the size of the parent grains of the iceberg, which is similar to that of some of the unaffected grains in the thin sections taken from the impacted surface. The average size of the parent grains is $10-15 \mathrm{~mm}$. The average size of grains within the fine-grain material is considerably less than $1 \mathrm{~mm}$ in diameter. During the microtoming process, it was noticed that the thin sections closer to the centre of the contact zone were more fragile than those near the edges.

A boundary between the fine-grained material and the parent grains was found in each of the thin sections taken from the impacted-surface slab. This boundary ran approximately parallel to the impacted surface at about $80 \mathrm{~mm}$ depth at the centre of the contact zone and $50 \mathrm{~mm}$ depth near the edge of the contact zone. Amongst the fine matrix of small grains, large parent-sized grains were found. Plate 13a shows an example of a large amount of fine-grained material found to exist between large unaffected grains.

A significant difference is seen in this main boundary parallel to the impacted surface when one compares thin sec- 
tions taken from near the centre with those taken from the outside edges of the contact zone. In thin sections taken near the centre, the boundary was not as distinctive, and lateral motion of grains along the boundary was not visible. In thin sections taken near the edge of the contact zone, the boundary was pronounced. The grains above the boundary appear to have shifted outwards in a lateral direction. Plate $14 \mathrm{a}-\mathrm{d}$ illustrates the movement of grains along the boundary in thin sections 1 and la taken from the impacted-surface slab. This is consistent with the extrusion process at the free edge of the high-pressure zone (see Fig. 1). In the magnified views of thin section la, shown in Plate $14 \mathrm{c}$ and d, the large, greencoloured grain in the bottom right quarter of the photograph can be seen to be offset along the boundary by approximately $10 \mathrm{~mm}$. This boundary may be considered to be similar to an extrusion plane with a "crack-like" boundary and the grains within being forced outwards towards a free surface.

The microstructural changes observed in the thin sections taken from the contact zone of the impacted iceberg suggest that the process of generating fine-grained material plays an important role in the deformation mechanisms during iceberg impacts. The increased depth of the zone of fine-grained material in the centre of the contact zone, as compared to the edges of the contact zone, is consistent with the configuration of a single high-pressure zone. The centre of the contact zone would correspond to the centre of the high-pressure zone where the confining pressure is higher. At the edges of the zone the confining pressure is significantly reduced, corresponding to a lower proportion of fine-grained material. Lateral motion of fine-grained material occurs as the confining pressure is suddenly released.

The process which generated the fine-grained material in the thin sections taken from the impacted surface is complex. This material is opaque in nature and appears to exist in bands which flow past unaffected grains. For example, thin section 8 from the impacted surface (see Plate 13a) shows mostly opaque fine-grained material which looks grey in colour when viewed between cross-polarized filters. The small grain structure may be caused by fine bands of microfractures which occurred within the grains. Under high confining pressure in the centre of the contact zone, it is expected that some pressure melting would occur during the impact. There is likely also to be dynamic recrystallization along grain boundaries and refreezing of the grains. See the discussion in Meglis and others (1999) on the occurrence of similar shear softening in other materials (e.g. mylonites in rocks).

In all thin sections taken from the impacted surface there appears to be a layer in which most of the microstructural changes in the ice occurred. These thin sections represent a "snapshot" of the ice after impact, and if the iceberg were to continue its interaction with the structure, the layer would progress further into the iceberg. The general layout of these layers may remain the same, changing only in space and time. What is observed in the thin sections taken from the impacted surface is a layer of highly rearranged and broken-down grains followed by parent grain material. Near the outer edges of the contact zone, the layer is narrower, but there is still a significant amount of fine-grained material contained in the layer (see, e.g., Plate 13d). It is also noted that in some cases thin sections taken from the edges of the contact zone have grains similar in size and orienta- tion to the parent grains at the top of the thin section close to the interaction surface.

Within the layer of most microstructural change found in the thin sections from the impacted-surface slab, there were parent-size grains amongst fine-grained material. Based on the orientation of groups of bubbles in the parentsize grains, some of these grains were found to have rotated during the impact process. The bubble orientation in the parent grains shown in thin sections taken from slab 2 is approximately $45^{\circ}$ from normal. The bubbles are seen as elongated elliptical shapes in Plate $14 \mathrm{c}$ and d, which gives the magnified view of thin section la from the impacted surface, and in Plate 13f, which gives the global view of thin section 3 from slab 2. The unaffected grains within the layer of most microstructural change with the same orientation as the parent grains (based on the bubble orientation) may have their $c$ axis in an orientation which makes them less likely to rotate or break down into smaller grains.

There is fine-grained material along the main boundary which divides the parent grains from the grains which have undergone the most microstructural change. This can be seen in Plate $14 \mathrm{a}$ and $\mathrm{b}$ which gives the magnified views of thin section 1 (CZl/IS/TSl). Fine-grained material along the main boundary line may be explained by the "chipping off" of grains as two or more grains are forced to slide along each other. The resulting fragments are expected to have been rounded through the process of slipping of the grains along the shear plane boundary. The fine-grained structure along the boundary may also help to facilitate further slip. This material may also be a result of nucleation of new grains.

Cracking is apparent throughout the thin sections along the impacted surface and in thin sections taken from slab 2 (see Plate 13e and f). There are randomly orientated cracks apparent throughout all the thin sections. These are believed to be pre-existing cracks because of their random orientation and their presence in the parent ice. Cracks which are out-of-plane may not be visible in the thin sections or may appear as thick grey lines as in thin section $\mathrm{CZ1} / \mathrm{S} 2 / \mathrm{TS} 3$ shown in Plate 13f. Randomly orientated cracks have been observed in naturally occurring iceberg ice. The cracks parallel to the impacted surface are assumed to be a direct result of the iceberg impact.

It is interesting to examine the cracking patterns readily visible in the selected photographs in Plate $15 \mathrm{a}$ and $\mathrm{b}$ taken under plain- and side-lighting conditions, respectively. As mentioned earlier, since these photographs were taken some time after the sections were made, only the pattern of cracking should be noted. Other details in these photographs will be neglected, since they are readily visible in the photographs of the same thin sections taken after the original thin-sectioning process. In Plate 15 , very fine dots in the order of $0.1 \mathrm{~mm}$ in diameter should be ignored. These are the result of sublimation and crystallization of the ice over time between the thin section and the glass plate. Cracks throughout thin section 8 and thin section ld from the impacted-surface slab are seen in Plate $15 \mathrm{a}$ and $\mathrm{b}$.

In Plate 15a, two large "rectangular-shaped" grains measuring approximately $35 \mathrm{~mm}$ across can be seen in the centre of thin section 8. These actually consist of a number of grains, as can be seen under cross-polarized filters shown in Plate 13a. The grains which make up each of the larger grains appear to have moved as a group. This movement has been determined through close examination of the crack lines through the two large grains. It appears that the two large 
grains were previously side by side and that the grain on the left has moved down relative to the other large grain. This movement of groups of grains suggests that there is some rearrangement and breakdown of grains within the central region of the contact zone which may eventually extrude out the edges of the contact zone as the ice advances.

In Plate 15b, many microcracks can be seen in thin section ld taken from the impacted-surface slab. The layer of highly modified microstructure above the main boundary is bright in colour due to the reflection of light from the many crack surfaces. The lateral movement of the grains along the boundary can easily be seen as crack lines are interrupted and offset.

\section{DISGUSSION}

As stated in Melanson and others (1999), the failure of ice in compression consists of fractures, with most of the load being carried through high-pressure zones that retain their integrity, at least for a short period of time. The pressure distribution over these zones has been observed to be parabolic in shape, with low confining pressures near the edges, and high pressures near the centre. Except at very low speeds of interaction, a distinct, narrow layer of highly damaged ice has invariably been observed to form adjacent to the structure. Examples of this have been given in the present paper, including the first examples to our knowledge of the formation of this layer in a real iceberg impact. Studies of the mechanics of the interaction process have been carried out (Jordaan and others, 1999), using the methods of damage theory. In this, state variables represent the prior stress history of ice at various locations, taking into account also the states of stress. In the simulations conducted, it was found that damage associated with microfracturing occurred first near the edges, then progressed inward. Softening associated with pressure effects then occurred near the centre and advanced towards the edge. This resulted in a reasonable simulation of layer formation, with sudden failure of the layer taking place when the two zones meet.

A comparison of the observations from the current thin sections and those from the small-scale laboratory tests described in Melanson and others (1999) and Meglis and others (1999) is given here. Figures have been chosen to illustrate several points. In comparing the current thin sections and the laboratory tests described in Melanson and others, the tests with higher strain rates were the main focus since they represented rates expected in full scale. Thin sections from the central region of the impacted surface of the contact zone described above were compared to the tests at $20 \mathrm{MPa}$ confining pressure, which may correspond to the centre of the contact zone (high-pressure zone). In particular, Plate 13a (thin section 8 taken from the central-region contact zone) was compared to Plate $2 \mathrm{~b}$. The microstructure in Plate $2 \mathrm{~b}$ is reasonably similar, indicating the field tests likely had strains in the $5 \%$ region. In Plate 2 , a substantial breakdown of grain-size can be seen with strains of $>2 \%$.

In comparing thin sections from the present paper and the laboratory-tested ice described in Meglis and others, example thin sections from the edge and from near the centre of the contact zone are compared to ice samples tested under conditions similar to those expected at the edge and at the centre of a high-pressure zone. Thin section 1 from the impacted-surface slab shown in Plate $13 \mathrm{~d}$ compares favour- ably with Plate $4 \mathrm{~b}$. The laboratory creep test was at a low confining pressure $\left(P_{\mathrm{c}}=5 \mathrm{MPa}\right)$ with a deviatoric stress of $15 \mathrm{MPa}$, a strain rate of $10^{-1}$ to $10^{-2} \mathrm{~s}^{-1}$ and a strain of about $10 \%$. This value of deviatoric stress is representative of the value expected towards the edge of the contact zone. Plate 4b shows extensive microcracking similar to that seen in Plate 15b (thin section ld taken from the edge of the contact zone with side lighting). At higher confining pressure corresponding to the centre of the contact zone, thin sections from Meglis and others showed the same breakdown of grains as seen in Plate 13a (thin section 8 taken from the centralregion contact zone). This is shown in Plate $7 \mathrm{a}$ which illustrates tests at $50 \mathrm{MPa}$ confinement, $15 \mathrm{MPa}$ applied deviatoric stress and a strain of about $4 \%$. The microstructure was similar to that seen in Melanson and others as discussed above for $20 \mathrm{MPa}$ confinement.

In summary, the results shown in the present thin section taken from the contact zones have microstructures consistent with confining pressure ranging from approximately $5 \mathrm{MPa}$ at the edges to about $20 \mathrm{MPa}$ near the centre. Highly damaged ice is likely to have the expected shear of approximately $15-25 \mathrm{MPa}$ and strains of $>4 \%$.

To allow comment to be made on the general observations represented in the thin sections taken from the contact zone as compared to results from some medium-scale indentation tests, measurements of the layer of modified microstructure due to the impact were made and a sketch was drawn (see Fig. 4a). The depth from the top of each thin section, corresponding to the impacted surface of the contact zone, to the boundary between the parent ice and the ice with changed microstructure was noted and indicated as points in Figure 4a. Each point corresponds to the average depth of the boundary and the approximate position of each thin section. A curve was drawn using the points as a guide to estimate the shape of the boundary. As mentioned earlier, the boundary was observed to have the greatest depth (approximately $80 \mathrm{~mm}$ into the ice) near the centre of the contact zone. Near the edges of the contact zone, the depth of the boundary from the impacted surface is approximately $50 \mathrm{~mm}$. The shape of the boundary is reasonably flat in the

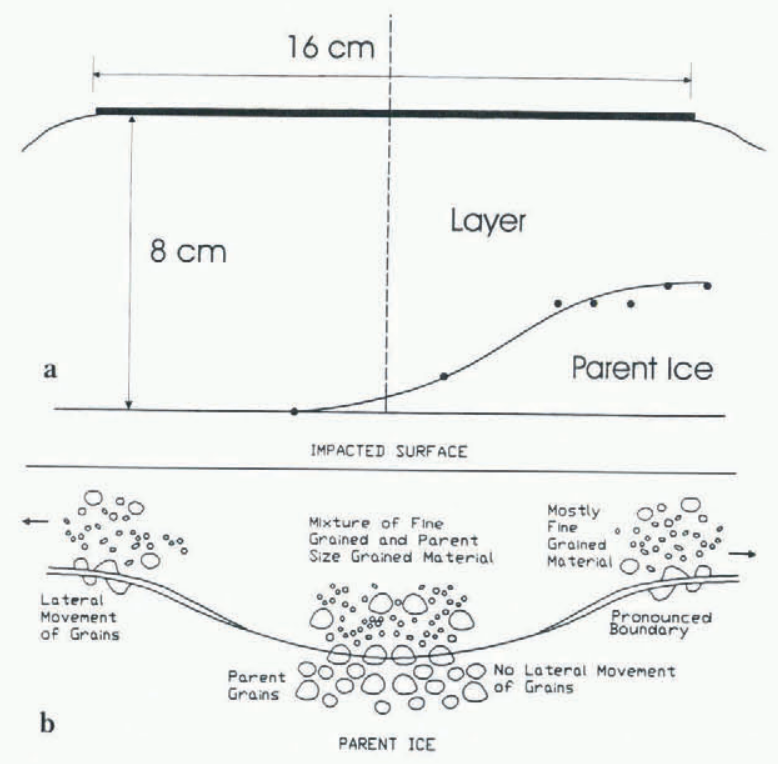

Fig. 4. Schematic drawings of profile of layer with most microstructural change. (a) Schematic of profile based on measurements of layer depth in thin sections. (b) Sketch of profile shape of the contact zone. 
middle, steep during the transition from the centre of the contact zone and level at the edge of the contact zone.

The boundary was more pronounced away from the centre of the contact zone, as illustrated in Figure $4 \mathrm{~b}$. The definition of the boundary appears to be linked to the amount of lateral movement of the grains. Grains near the centre of the contact zone had little lateral movement. At the edges of the contact zone, the lateral motion of grains was significant, and offset of many individual grains was seen above the boundary. Fine-grained material surrounding unaffected parent-size grains was observed in the layer both near the centre and near the edge of the contact zone. The centre of the contact zone is considered to be the approximate centre of a high-pressure zone, with lower pressures at the edges where extrusion of fine ice particles occurs.

The thin sections were compared to the results of medium-scale indentation tests in the Arctic Ocean in 1989 (Frederking and others, 1990), where crushing and extrusion processes and high-pressure zones were evident. A photograph of the ice face after an indentation test from Frederking and others (1990) is included in Plate 16. Plate 16a shows the contact face with a view of the crushed layer. Plate $16 \mathrm{~b}$ shows a thin section taken from the area indicated by a box in Plate 16a. In Plate 16a, the shape of the layer is clearly shown. The central region of the layer, which is included in the area indicated by the box, corresponds to the blue zone of the contact face. On both sides of this central area, a layer of opaque white ice was observed continuing to the edges of the contact face. The ice in this zone has been crushed and pulverized into small grains. Near the centre, most of the large grains remain, but with incipient damage on the grain boundaries and in some grains (Frederking and others, 1990). It was noted by Frederking and others (1990) that the edge of the contact zone was soft, while the centre was quite solid even in areas where the ice had been highly pulverized. This finding is in agreement with the microstructure of the ice sample shown in Plate 16b. The layer of highly pulverized ice can be seen on either side of the central region. The boundary between the fine grains and the parent grains is very distinct away from the central region, particularly at the bottom right of Plate 16b. In the centre, there are mostly parent-size grains, but the orientation of the grains is not random (as in undamaged ice) and some change in microstructure is apparent. Results of the indentation tests indicate that at higher indentation rates, which would more closely represent iceberg impacts, a relatively thin layer of crushed and damaged ice with extrusion of fine particles was observed. The thickness of the layer was found to vary throughout the layer, but a narrow band of relatively undamaged ice usually in the central part of the contact zone was observed after each test. In the central part of the contact zone, measurements of local ice pressures were about three times higher than the average pressure over the entire contact face (Frederking and others, 1990).

Plate 17a, taken from Meaney (1992), shows a horizontal thick section taken from an ice face after impact with a flat rigid indentor (test denoted TFR-01) at Hobson's Choice ice island in 1990. Meaney (1992) stated that although the centre of the horizontal thick section was damaged, a discrete crushed layer did not exist. The crushed layer shown in Plate 17a had an average thickness of $57 \mathrm{~mm}$ and a maximum thickness of $155 \mathrm{~mm}$, and was found to increase in thickness toward the perimeter of the ice face. A sketch of the zones observed on the test face, and the corresponding plan view depicting the layer, is given in Plate 17b from Meaney (1992) and Meaney and others (1996). This sketch shows the distinct boundary away from the central region and the lack of a distinct boundary in the centre. The layer which extends to the edges of the test face is a zone of pulverized fine-grained ice. Segments near the edges of the contact face which broke off during the test are shown as spalled areas in Plate 17b. Density measurements along the crushed layer showed that the density increased towards the centre of the crushed layer. The substantially greater confining pressure in the centre of the indentor was thought to contribute to this finding. The crushed layer was found to contain large $(>25 \mathrm{~mm})$, relatively undamaged particles surrounded by a matrix of pulverized ice, suggesting a grinding action as particles slide and rotate during extrusion (Meaney, 1992). Meaney also noted the differences produced by the use of different shapes of indentors. The wedge indentors produced a higher degree of spalling than the flat indentors, and the layer of crushed ice produced with the wedge indentor was indicative of the lower confinement present.

A section through the impacted surface after a sphericalhead indentation test at Hobson's Choice ice island in 1989 is presented in Figure 5 for comparison to the present set of thin sections. The rigid spherical indentor used had an area of $0.8 \mathrm{~m}^{2}$ with a $1.28 \mathrm{~m}$ radius of curvature and $1 \mathrm{~m}$ overall diameter. Figure 5 shows the same type of crushed layer containing fine-grained material. A pronounced boundary is seen some distance away from the centre of the contact zone. The central region contains a mixture of fine grains and parent-sized grains and extends some way into the ice. This is most likely due to the shape of the spherical indentor and the confinement at the head of the indentor. In discussions of the indentation tests in Jordaan and others (1990), the ice within the layer is stated to have a lower density than the parent ice. The sintered nature of the ice after the tests was discussed and the importance of pressure melting in the deformation process was noted. Observations were found to agree with earlier observations from drop-ball tests (Kheysin and Cherepanov, 1970).

Similarities found in the various indentation tests discussed and in the present thin sections are the formation of a layer of fine-grained material and the pronounced boundary towards the edges of the contact zone. In all tests, the central region showed changes in microstructure within the ice but no pronounced boundary. The difference in the depth of changes in microstructure in the central region was noted for the various examples mentioned. In the indentation tests, the central region was a thin band. In the present thin sections taken from the contact zone, the central region of microstuctural change in the ice extends slightly further into the sample. In all tests, there was evidence of extensive cracking, extrusion of small crushed ice particles and pressure melting.

Based on the observations made of the microstructural changes in the impacted-iceberg contact zone, a number of suggestions for further work are presented. It is important to use iceberg ice in future small-scale testing and to conduct tests under loading and confinement conditions similar to those expected in iceberg-structure interaction (e.g. test durations of similar length to iceberg impacts). Comparisons of the microstructural changes under different loading and confinement conditions have given insights into the deformation mechanisms occurring in the ice. See Melanson and others (1999) and Meglis and others (1999) for recent labora- 


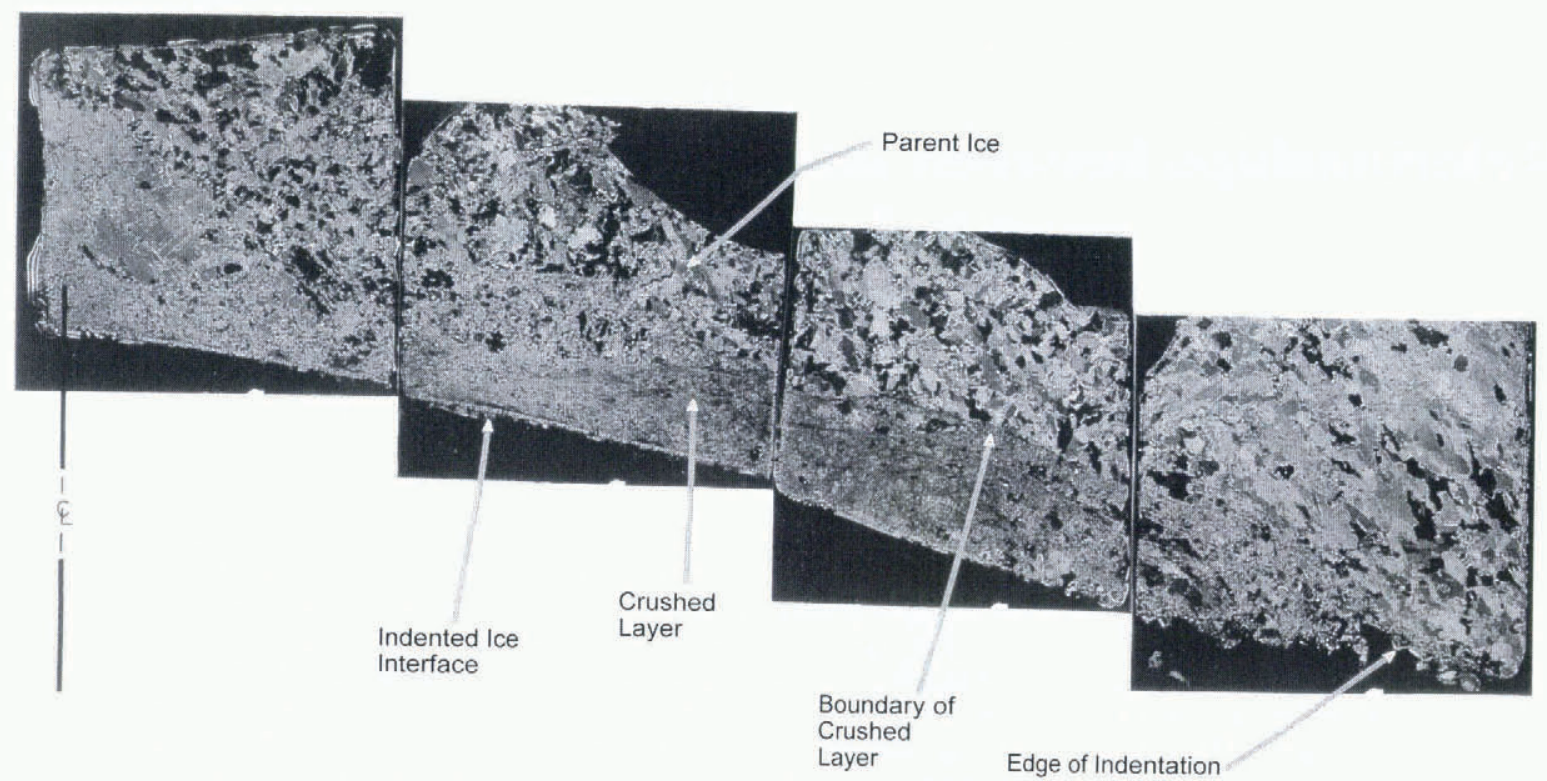
Fig. 5. Section across crushed layer after spherical-head indentation test, Hobson's Choice ice island, 1989 (photo taken by E.
Stander and used with permission).

tory test programs conducted at Memorial University. Continued laboratory testing in these areas along with further full-scale testing would help to improve understanding of the processes occurring in the ice during iceberg impacts.

\section{GONCLUDING REMARKS}

A few of the highlights of the observations of the microstructure found in the contact zone are noteworthy. A layer of ice with highly modified microstructure was seen in the thin sections taken from the impacted surface. The boundary between the ice with modified microstructure and the parent ice was found to be more pronounced away from the central region of the contact zone. Fine-grained material in the layer on either side of the central region was found to move in a lateral direction toward the edges of the contact zone. These findings are consistent with the concept of a high-pressure zone with higher pressure in the centre and lower pressure at the edges as ice is extruded. Cracking was evident throughout the thin sections in various directions. Cracking parallel to the impacted surface is thought to be a direct result of the iceberg-impact process and may be due to unloading. The findings concerning the present thin sections were similar to those of previous medium-scale indentation tests.

\section{ACKNOWLEDGEMENTS}

The authors would like to acknowledge the support obtained through the National Energy Board, Canada. G. B. Crocker and G. M. English of C-CORE provided valuable information for the project, and their enthusiastic cooperation is gratefully acknowledged. The authors acknowledge the use of the facilities at the Institute for Marine Dynamics, National Research Council of Canada. The assistance of B. M. Stone, J. F. Sweeney, I. L. Meglis and P. M. Melanson was appreciated. Discussions with R. E. Gagnon and S. J. Jones of the Institute for Marine Dynamics were also helpful. The authors thank E. Stander for permission to use Figure 5. This work was supported in part by the Natural Sciences and Engineering Research Council and the Canada-Newfoundland Offshore Development Fund. The authors thank the reviewers for their constructive and useful comments.

\section{REFERENCES}

Crocker, G. 1995. Iceberg impact experiment. C-CORE. Newes, 20 (3), 10-12. Crocker, G. B., K. R. Croasdale, R. F. McKenna, G. M. English, J. Guzzwell and S. Bruneau. 1997. C-CORE iceberg impact experiment - Phase 2, final report. St John's, Nfld, Memorial University of Newfoundland. Centre for Cold Ocean Resources Engineering. (C-CORE Contract Report 96-Cl6.)

Frederking, R., I. J. Jordaan and J. S. McCallum. 1990. Field tests of ice indentation at medium scale, Hobson's Choice Ice Island, 1989. In IAHR Symposium on Ice, Espoo, Finland, August 20-24, 1990. Proceedings. Vol. 2. Espoo, Helsinki University of Téchnology, 931-944.

Jordaan, I. J. and 6 others. 1990. Development of new ice load models. St John's, Nfld, Memorial University of Newfoundland. Centre for Cold Ocean Resources Engineering (C-CORE).

Jordaan, I. J., D. G. Matskevitch and I. L. Meglis. In press. Disintegration of ice under fast compressive loading. Int. J. Fract., 97 (1-4), 279-300.

Kheysin, D. Ye. and N.V. Cherepanov. 1970. Izmeneniye struktury l'da v zone udara tverdogo tela o poverkhnost' ledyanogo pokrova [Change of ice structure in the zone of impact of a solid body against the ice cover surface]. Probl. Arkt. Antarkt. 34, 79-84.

Meaney, R. B. 1992. Localized crushing in ice-structure interaction. (M.Eng thesis, Memorial University of Newfoundland.)

Meaney, R., I. J. Jordaan and J. Xiao. 1996. Analysis of medium scale iceindentation tests. Cold Reg. Sci. Technol., 24 (3), 279-287.

Meglis, I. L., P. M. Melanson and I.J. Jordaan. 1999. Microstructural change in ice: II. Creep behavior under triaxial stress conditions. F. Glaciol., 45 (15l), 438-448.

Melanson, P. M., I. L. Meglis, I. J. Jordaan and B. M. Stone. 1999. Microstructural change in ice: I. Constant-deformation-rate tests under triaxial stress conditions. f. Glaciol., 45 (151), 417-422.

Memorial University of Newfoundland. 1996. Canadian Offshore Design for Ice Environment (CODIE) second annual report. St John's, Nfld, Memorial University of Newfoundland. Ocean Engineering Research Centre. 